In view of applications, let us remark that every enumerable set (for example the set of rational or algebraic numbers $a, b$ ) of substitutions $S_{a b}$ is non-normal, that is to say, it is impossible, without using particular hypothesis on the $a_{i j}$, to deduce the convergence of $\bar{F}$ (except for $x=y=0$ ) and the analy ticity of $F$ from the convergence of the series $F_{a b}(t)$.

\title{
BIBLIOGRAPHY
}

1. M. A. Zorn, Bull. Amer. Math. Soc. vol. 53 (1947) pp. 791-792

2. Rimhak Ree, Bull. Amer. Math. Soc. vol. 55 (1949) pp. 575-576.

3. Evans, Monatshefte für Mathematik vol. 43 (1936) pp. 419-424.

4. G. P6lya and G. Szegö, J. Reine Angew. Math. vol. 165 (1931) pp. 4-49.

5. T. Rado, Subharmonic functions, Ergebnisse der Mathematik und ihre Grenzgebiete, vol. $5,1,1937$.

6. M. Brelot, C. R. Acad. Sci. Paris vol. 207 (1938) p. 836.

7. P. Lelong, C. R. Acad. Sci. Paris vol. 210 (1940) p. 471.

8. - Ann. Ecole Norm. vol. 58 (1941) pp. 83-177.

9. H. Cartan, Bull. Soc. Math. France vol. 73 (1945) pp. 74-106.

FACULte DES SCIENCES DE LiLle

\section{NOTE ON A SERIES OF PRODUCTS OF THREE LEGENDRE POLYNOMIALS}

\author{
JOHN P. VINTI
}

Let $x, y$, and $z$ be real variables and $P_{l}$ the Legendre polynomial of order $l$. In this note we shall prove the following theorem:

$$
\begin{aligned}
& \sum_{l=0}^{\infty}\left(l+\frac{1}{2}\right) P_{l}(x) P_{l}(y) P_{l}(z)=\pi^{-1} g^{-1 / 2} \quad(g>0) \\
& =0 \quad(g<0) \\
& (-1<x<1,-1<y<1,-1<z<1) \text {, }
\end{aligned}
$$

where

$$
g(x, y, z) \equiv 1-x^{2}-y^{2}-z^{2}+2 x y z .
$$

Furthermore, if $T_{+}$be the region of $x, y, z$ space, as above delimited, for which $g>0$, and $T$ - be the region for which $g<0$, the convergence is uniform with respect to any one of the variables $x, y$, or $z$, taken singly, that is, throughout any closed interval along which only one of the variables changes and which is interior either to $T_{+}$or to $T_{-}$.

Received by the editors November 2, 1949. 
Before proving the theorem, it is of interest to state the geometrical significance of the function $g(x, y, z)$. The condition $g>0$ is necessary and sufficient for the existence of a spherical triangle of sides $\cos ^{-1} x, \cos ^{-1} y, \cos ^{-1} z$, that is, for the existence of a trihedral angle with face angles $\cos ^{-1} x, \cos ^{-1} y, \cos ^{-1} z$. Then $g^{1 / 2}$ is equal to the volume of a parallelepiped of unit sides, possessing such a trihedral angle at one vertex.

To prove the theorem, consider the function $f(x, y, z)$, defined as follows:

$$
\begin{array}{cr}
f(x, y, z) \equiv \pi^{-1} g^{-1 / 2} & (g>0) \\
\equiv 0 & (g \leqq 0) \\
(-1 \leqq x \leqq 1,-1 \leqq y \leqq 1,-1 \leqq z \leqq 1) .
\end{array}
$$

We proceed to expand $f$ in the Legendre series

$$
f(x, y, z)=\sum_{l=0}^{\infty}\left(l+\frac{1}{2}\right) C_{l}(y, z) P_{l}(x)
$$

where

$$
C_{l}(y, z) \equiv \int_{-1}^{1} P_{l}(x) f(x, y, z) d x
$$

Hobson $^{1}$ has given sufficient conditions for the validity of such an expansion. For the present purpose they may be specialized as follows (since $f$ is continuous in any region interior to $T_{+}$or interior to $\left.T_{-}\right)$: Let

$$
J \equiv \int_{-1}^{1}\left(1-x^{2}\right)^{-1 / 4}|f(x, y, z)| d x .
$$

If $J$ exists and if $\left(\alpha_{1}, \alpha_{2}\right)$ is a closed subinterval given by $-1<\alpha_{1}$ $\leqq x \leqq \alpha_{2}<1$ interior either to $T_{+}$or to $T_{-}$, then (3) converges to $f(x, y, z)$ and converges uniformly with respect to $x$ throughout the closed interval $\left(\alpha_{1}, \alpha_{2}\right)$.

To prove the theorem we thus need prove only that $J$ exists and that $C_{l}(y, z)=P_{l}(y) P_{l}(z)$. Repetition of the argument, with the aid of Legendre expansions in $P_{l}(y)$ or in $P_{l}(z)$, will then show that, in any closed region interior to $T_{+}$or interior to $T_{-}$, the convergence of (1) is uniform with respect to any one of the variables $x, y$, or $z$, taken singly, that is, throughout any closed interval along which only one

${ }^{1} \mathrm{E}$. W. Hobson, The theory of spherical and ellipsoidal harmonics, Cambridge University Press, 1931, p. 329 ff. 
of the variables changes and which is interior to $T_{+}$or to $T_{-}$. (It is my conjecture that the convergence of (1) is uniform throughout the whole of any closed region interior either to $T_{+}$or to $T_{-}$.)

We next develop some relations that will be needed.

$$
\begin{aligned}
g(x, y, z) & \equiv 1-x^{2}-y^{2}-z^{2}+2 x y z \\
& \equiv\left(1-y^{2}\right)\left(1-z^{2}\right)-(x-y z)^{2} .
\end{aligned}
$$

If either $y$ or $z$ equals \pm 1 , then $g \leqq 0$ and thus $f=0$ over the whole interval $-1 \leqq x \leqq 1$. In this case $J=0$ and the Legendre series, although valid, gives only the triviality $0=0$.

We now restrict consideration to $-1<y<1,-1<z<1$. In this case $\left(1-y^{2}\right)\left(1-z^{2}\right)>0$, so that the following condition is necessary and sufficient for $g>0$ :

$$
(x-y z)^{2}<\left(1-y^{2}\right)\left(1-z^{2}\right) .
$$

If we now define a real number $\phi$ by

$$
\phi \equiv \cos ^{-1}\left[(x-y z)\left(1-y^{2}\right)^{-1 / 2}\left(1-z^{2}\right)^{-1 / 2}\right]
$$

so that

$$
x=y z+\left(1-y^{2}\right)^{1 / 2}\left(1-z^{2}\right)^{1 / 2} \cos \phi,
$$

then the following conditions are also necessary and sufficient for $g>0$ :

$$
-1<\cos \phi<1
$$

or

$$
x_{1}(y, z)<x<x_{2}(y, z)
$$

where

$$
x_{1}(y, z) \equiv y z-\left(1-y^{2}\right)^{1 / 2}\left(1-z^{2}\right)^{1 / 2}
$$

and

$$
x_{2}(y, z) \equiv y z+\left(1-y^{2}\right)^{1 / 2}\left(1-z^{2}\right)^{1 / 2}
$$

are the values of $x$ satisfying $g(x, y, z)=0$.

Since

$$
x_{1}^{2}=1-\left[y\left(1-z^{2}\right)^{1 / 2}+z\left(1-y^{2}\right)^{1 / 2}\right]^{2}
$$

and 


$$
x_{2}^{2}=1-\left[y\left(1-z^{2}\right)^{1 / 2}-z\left(1-y^{2}\right)^{1 / 2}\right]^{2},
$$

it follows that $-1 \leqq x_{1} \leqq 1$ and $-1 \leqq x_{2} \leqq 1$.

The existence of $J$ is not obvious, because of the infinite discontinuities of $f$ at $x_{1}$ and $x_{2}$. To prove its existence we note that

$$
\begin{array}{rlrl}
|f(x, y, z)|=f(x, y, z) & =0 & \left(-1 \leqq x \leqq x_{1}\right) \\
& =\pi^{-1} g^{-1 / 2} & & \left(x_{1}<x<x_{2}\right) \\
& =0 & & \left(x_{2} \leqq x \leqq 1\right) .
\end{array}
$$

Then

$$
J=\frac{1}{\pi} \int_{x_{1}}^{x_{2}}\left(1-x^{2}\right)^{-1 / 4} g^{-1 / 2} d x .
$$

We now replace $x$ by $\phi$ as integration variable, the corresponding limits on $\phi$ being from $\pi$ to 0 . Then, since $g^{1 / 2}=\left(1-y^{2}\right)^{1 / 2}\left(1-z^{2}\right)^{1 / 2}$ $\cdot \sin \phi$ in $0 \leqq \phi \leqq \pi$, we have

$$
J=\frac{1}{\pi} \int_{0}^{\pi}\left(1-x^{2}\right)^{-1 / 4} d \phi .
$$

In (11.1), $-1 \leqq x_{1} \leqq x \leqq x_{2} \leqq 1$, so that $x^{2}$ can equal 1 only if $x_{1}^{2}=1$ or $x_{2}^{2}=1$. From (9.3) and (9.4) it is seen that $x_{1}^{2}=1$ only for $y=-z$ and $x_{2}^{2}=1$ only for $y=z$. Thus $J$ exists if $y \neq \pm z$.

For each of the remaining cases, $y= \pm z$, we find easily

$$
J=\frac{2}{\pi}|a|^{-1 / 2} \int_{0}^{\pi / 2}(\sin \theta)^{-1 / 2}\left(2-a^{2} \sin ^{2} \theta\right)^{-1 / 4} d \theta
$$

where $a^{2} \equiv 2\left(1-y^{2}\right)$, so that $0<a^{2} \leqq 2$ for $-1<y<1$. Since $a^{2} \leqq 2$, we find that the

$$
\begin{array}{r}
\text { integrand } \leqq \frac{2^{1 / 2}}{\pi}\left(1-y^{2}\right)^{-1 / 4}\left(1+\pi^{2} / 4\right)^{1 / 2} \theta^{-1 / 2}(\pi / 2-\theta)^{-1 / 2} \\
(0 \leqq \theta \leqq \pi / 2) .
\end{array}
$$

But $\int_{0}^{\pi / 2} \theta^{-1 / 2}(\pi / 2-\theta)^{-1 / 2} d \theta=\pi$, so that $J$ exists also for $y^{2}=z^{2} \neq 1$. Note that for $y^{2}=z^{2}=1$ the above formula (11.2) for $J$ is not applicable, $J$ vanishing in such a case and the Legendre series giving only a trivial result.

It remains only to prove that $C_{l}(y, z)=P_{l}(y) P_{l}(z)$. We have

$$
C_{l}(y, z)=\int_{-1}^{1} P_{l}(x) f(x, y, z) d x=\frac{1}{\pi} \int_{x_{1}}^{x_{2}} P_{l}(x) g^{-1 / 2} d x .
$$

With $\phi$ as integration variable, 


$$
\begin{aligned}
C_{l}(y, z) & =\frac{1}{\pi} \int_{0}^{\pi} P_{l}\left[y z+\left(1-y^{2}\right)^{1 / 2}\left(1-z^{2}\right)^{1 / 2} \cos \phi\right] d \phi \\
& =\frac{1}{2 \pi} \int_{0}^{2 \pi} P_{l}\left[y z+\left(1-y^{2}\right)^{1 / 2}\left(1-z^{2}\right)^{1 / 2} \cos \phi\right] d \phi
\end{aligned}
$$

which is immediately seen, from the addition theorem of spherical harmonics, to equal $P_{l}(y) P_{l}(z)$. This completes the proof of the theorem.

At any point $(x, y, z)$ for which $g(x, y, z)=0$ the function $f$ has an infinite discontinuity and the Legendre series is not valid. The theorem, therefore, does not apply at such a point.

We now consider the end values $x= \pm 1$, with $-1<y<1$ and $-1<z$ $<1$. Convergence of the Legendre series for $f(x)$ at $x= \pm 1$ is, normally, to the values $f(1-0)$ or $f(-1+0)$, respectively, when it can occur. Hobson (loc. cit.) has established sufficient conditions for convergence or nonconvergence to these values at the end points. From his criteria it is evident that the present Legendre series in $P_{l}(x)$, and thus (1), will not converge to the orthodox values at $x= \pm 1$; by expanding $f(x, y, z)$ in terms of $P_{l}(y)$ or $P_{l}(z)$ we see similarly that (1) will not so converge at $y= \pm 1$ or $z= \pm 1$. These conclusions are in agreement with the well known Christoffel relation for the partial sum of (1) when $z=1$, for example. In that case

$$
\sum_{l=0}^{n}\left(l+\frac{1}{2}\right) P_{l}(x) P_{l}(y)=\frac{n+1}{2(x-y)}\left[P_{n+1}(x) P_{n}(y)-P_{n}(x) P_{n+1}(y)\right],
$$

which does not approach a limit as $n$ increases.

It is a pleasure to acknowledge stimulating discussions with Dr. A.

S. Galbraith and Dr. W. C. Taylor.

Ballistic Research laboratories, Aberdeen Proving Ground 